\title{
Social Control of Herd Animals by Integration of Artificially Controlled Congeners
}

\author{
Nikolaus Correll, Mac Schwager, and Daniela Rus \\ Computer Science and Artificial Intelligence Laboratory \\ Massachusetts Institute of Technology \\ Cambridge, MA 02139
}

\begin{abstract}
We study social control of a cow herd in which some of the animals are controlled by a sensing and actuation device mounted on the cow. The control is social in that it aims at exploiting the existing gregarious behavior of the animals, rather than controlling each individual directly. As a case study we consider the open-loop control of the herd's position using location-dependent stimuli. We propose a hybrid dynamical model for capturing the dynamics of the animals during periods of grazing and periods of stress. We assume that stress can either be induced by the sensing and actuation device or by social amplification due to observing/overhearing nearby stressed congeners. The dynamics of the grazing part of the proposed model have been calibrated using experimental data from 10 free-ranging cows, and various assumptions on the animal behavior under stress are investigated by a parameter sweep on the hybrid model. Results show that the gregarious behavior of the animals must be increased during stress for control by undirected stimuli to be successful. We also show that the presence of social amplification of stress allows for robust, low-stress control by controlling only a fraction of the herd.
\end{abstract}

\section{Introduction}

We wish to study the potential of low-stress managing a cow herd by exploiting the cows's innate gregarious behavior using a small number of controlled animals. The idea of controlling herd animals using robotic agents [1] or by devices mounted on the animals ("smart collars") [2-5] bears great potential for revolutionizing animal husbandry. Cows seem to be particularly well suited for this endeavor as keeping them in their natural environment is labor intensive and costly.

Recently, the idea of controlling groups of animals by integrating artificially controlled agents into the animal society and leveraging the natural effects of gregarious behavior has been brought forward in [6]. In [6], cockroaches were presented with a dark and a bright shelter in a circular arena, and usually aggregate under the dark shelter, which they prefer. Miniature robots [7] were then impregnated with cockroach pheromones and integrated into the cockroach swarm. Unlike the cockroaches, the robots were programmed with a preference for the bright shelter. As shelter selection was shown to be a social decision, the robots could thus bias the choice of the cockroaches. 
Similarly for husbandry, we wish to identify social behavior in farm animals that can be exploited by integration of a small number of controlled animals into the herd, instead of controlling each individual separately. In this paper, we approach this goal for a cow herd by using a hybrid model of the herd dynamics. The model has been developed based on physical principles and calibrated using a system identification process based on data collected from 10 cows on the United States Department of Agriculture, Agricultural Research Services (USDA-ARS) experimental ranch in Las Cruces, New Mexico, USA [8].

We hypothesize that two social effects will enable the control of the herd. The first social effect is that when an animal is stressed, its gregarious tendencies are increased, thus drawing it to the center of the herd. The second social effect (which is backedup by observations in [9]) is that a stressed animal may pass on its stressed state to its neighbors. Stress induced by a device mounted on the animal, e.g. due to an aural stimulus, does not only alter the behavior of the animal itself, but also affects nearby animals, which see or hear a stressed congener. This mechanism bears the potential for amplifying the effect of external stimuli provided to only a few cows. In this paper we extend the model from [8] to include these two social effects. The increased agreggation tendency of stressed animals is parameterized by a constant factor, and the propagation of stress from one animal to another is modeled by a radius of detection and a time constant allowing for temporal stress decay. The resulting dynamical model is a hybrid system with two modes: stressed and grazing.

Using extensive simulations of the hybrid dynamical model, we study the potential for social herding by employing a mobile virtual fence [9], which induces stress in those animals that are outside the fenced area and wear sensing and actuation devices. The mechanism that we rely on to control the herd is quite different from those described in previous virtual fencing studies, however. We do not give the animal any directional information from the cue itself. That is to say, when an animal is cued in our model, it does not know where it is "supposed" to go. It simply reacts in a stressed manner, which causes it to draw closer to the center of the herd while propagating its stressed state to its neighbors. In contrast, previous approaches have used graduated cuing intensity or stereo cuing to give directional information to animals [4]. Directional cuing may be useful, especially for animals that have been trained to interpret the directional cues. However, it has been noted in human subjects that it is difficult to detect meaningful directional information from such directional cuing [9]. The results of our simulation study imply that a herd can still be managed effectively with a control system that gives no directional information by leveraging the animals' natural gregarious instincts. Our results show both the potential and the conditions for social control of a cow herd. Furthermore, we provide an agenda for further field experiments, which are required to identify the parameters of the extended model proposed in this paper and validate our approach.

\section{Data-Collection Experiments}

Cows were equipped with a small light-weight box for data collection during field experiments [8], see Figure 1. The box contains electronics for recording the GPS location 
of the animal as well as other sensor data (e.g. position of the head, body orientation) and environmental data (e.g. temperature). The box also contains electronics for networking the herd. The sensing box was used for collecting trajectory data from 10
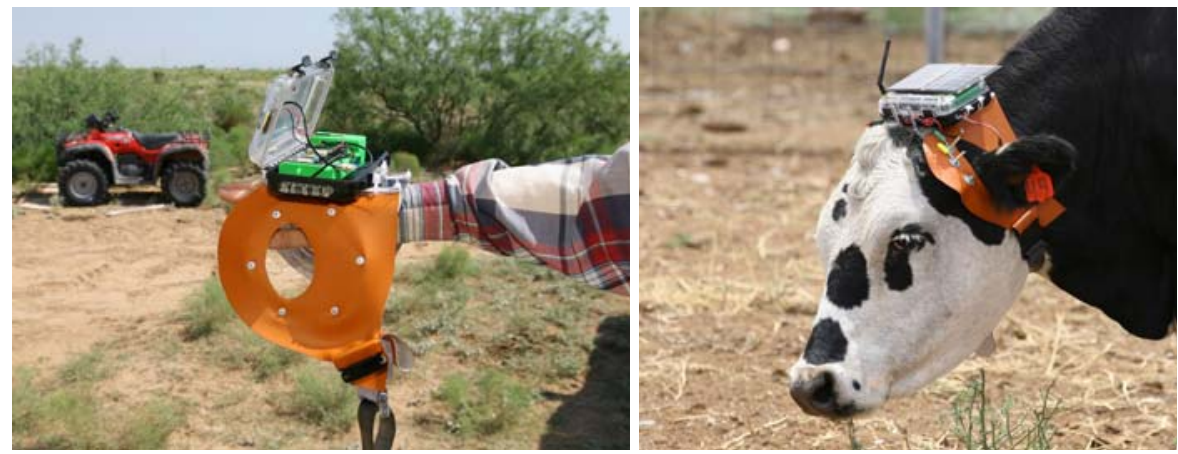

Fig. 1. The sensing and actuation box [8] is roughly $21.5 \mathrm{~cm} \times 12.0 \mathrm{~cm} \times 5.5 \mathrm{~cm}$ and weighs around $1 \mathrm{~kg}$. It is equipped with a GPS receiver, wireless networking features, and a suit of sensing and actuation capabilities. The Lithium-Ion batteries and solar panel allow for indefinite operation under normal conditions. The sensor box is mounted to the head of a cow with a leather strap designed to use the cow's ears to keep the box in position. Pictures courtesy of I. Vasilescu.

free-ranging mature cows (Hereford and Hereford $\times$ Brangus genetics) in a 466ha area on the USDA-ARS's Jornada Experimental Range (JER). This site has an undulating topography of predominantly sandy soil, populated with grasses and shrubs. Trajectory data were collected over 3 days at a rate of $1 \mathrm{~Hz}$. Parts from these data were then used for calibrating the parameters for the model used in this paper. The calibration process as well as the animal experiments are described in more detail in [8]. For future experiments, the box provides a two-tier animal control system consisting of a set of speakers for applying arbitrary, differential sound stimuli and a set of electrodes that enable the application of differential electric shock. The animal control system was not used during the collection of the data used in this paper.

\section{Model}

In [8] we developed a linear-in-parameter model that provides good qualitative and quantitative agreement with various individual and collective metrics when used for simulating grazing cows. In this paper, we introduce a hybrid model, whose single discrete state variable corresponds to the behavioral modes grazing and stressed. The dynamics in each behavioral mode only differ by an increased motivation to aggregate and increased speed of the animal. The dynamics in both grazing and stressed behavioral modes are governed by two naturally distinct mechanisms. First, each agent is given internal dynamics to enforce the constrains of Newton's laws. Second, a force is applied to each agent from its interaction with each of the other agents in the group. All remaining effects are modeled as a white noise process. 


\subsection{Individual Agent Dynamics}

Given a group of $m$ agents, every individual is modeled by a hybrid model consisting of a continuous dynamical part, describing position and velocity of an individual, and a discrete part modeling the individual's behavioral mode. This model is illustrated by the Finite State Machine depicted in Figure 2, left.
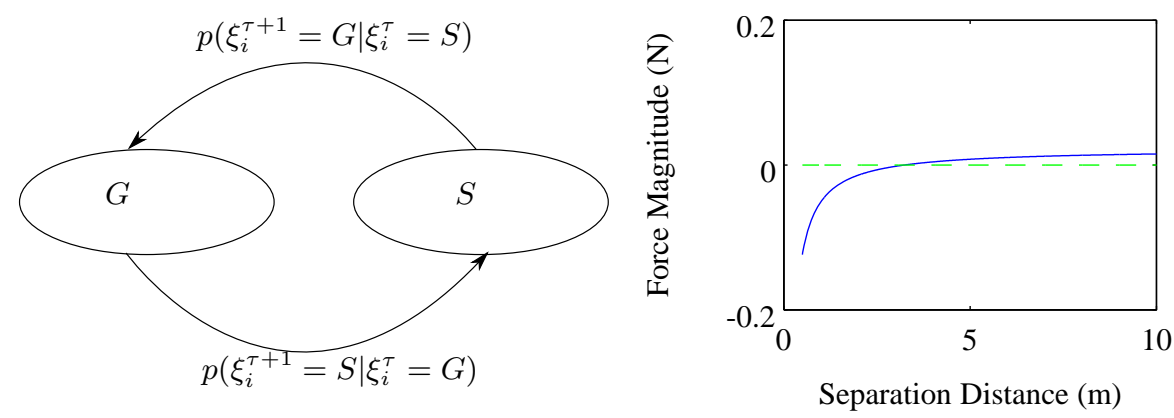

Fig. 2. Left: Probabilistic Finite State Machine modeling an agent's behavioral mode and its transitions. Each behavioral mode has its own linear-in-parameter dynamics. Right: The magnitude of the agent-to-agent interaction force for $\theta_{1}=.0225, \theta_{2}=.0732$ and $\alpha=1$, which corresponds to the average value measured between 10 cows.

We distinguish between two different behavioral modes, which determine the dynamics of the agent. Agent $i$ 's behavioral mode at any time $\tau$ is given by $\xi_{i}^{\tau} \in\{G, S\}$, which corresponds to grazing $\left(\xi_{i}^{\tau}=G\right)$ or stressed behavior $\left(\xi_{i}^{\tau}=S\right)$. We define the behavior mode transition rules as

$$
p\left(\xi_{i}^{\tau+1}=S \mid \xi_{i}^{\tau}=G\right)= \begin{cases}1 & \text { if } \mathcal{N}_{S, i}^{\tau}(R)>\mathcal{N}_{G, i}^{\tau}(R) \vee u_{i}^{\tau}=1, \\ 0 & \text { otherwise }\end{cases}
$$

where $\mathcal{N}_{S, i}^{\tau}(R)$ and $\mathcal{N}_{G, i}^{\tau}(R)$ are the number of agents in behavioral mode $\xi_{i}^{\tau}=S$ or $\xi_{i}^{\tau}=G$, respectively, within a radius of $R$ around agent $i$. Also, $u_{i}^{\tau} \in\{0,1\}$ is the binary control input to the cow, so that $u_{i}^{\tau}=0$ corresponds to no control stimuli at time $\tau$, while $u_{i}^{\tau}=1$ when the animal is receiving a stimuli at time $\tau$. Similarly, we define

$$
p\left(\xi_{i}^{\tau+1}=G \mid \xi_{i}^{\tau}=S\right)=\frac{1}{T}
$$

where $T$ is a time constant modeling stress decay.

The dynamics of agent $i \in\{1, \ldots, m\}$ can be written in state-space, difference equation form as

$$
x_{i}^{\tau+1}=\left[\begin{array}{cccc}
1 & 0 & \Delta t & 0 \\
0 & 1 & 0 & \Delta t \\
0 & 0 & a_{i} & 0 \\
0 & 0 & 0 & a_{i}
\end{array}\right] x_{i}^{\tau}+\left[\begin{array}{ll}
0 & 0 \\
0 & 0 \\
1 & 0 \\
0 & 1
\end{array}\right]\left(\frac{1}{m} \sum_{j=1, j \neq i}^{m} f_{i j}\left(p_{i}^{\tau}, p_{j}^{\tau}, \xi_{i}^{\tau}\right)+w_{i}^{\tau}\left(\xi_{i}^{\tau}\right)\right)
$$


The state of agent $i$ is given by the vector $x_{i}^{\tau}$, consisting of its East position, North position, Eastern component of velocity, Northern component of velocity. An agent's position is thus given by, $p_{i}^{\tau}=\left[\begin{array}{ll}e_{i}^{\tau} & n_{i}^{\tau}\end{array}\right]^{T}$. The time step $\Delta t$ is given by $t^{\tau+1}-t^{\tau}$, and we assume it is constant for all $\tau$. The term $a_{i}$ represents damping, $a_{i}=1$ for zero damping, and $\left|a_{i}\right|<1$ for stable systems. The function $f_{i j}\left(p_{i}^{\tau}, p_{j}^{\tau}, \xi_{i}^{\tau}\right)$ determines the coupling force applied by agent $j$ to agent $i$ as a function of the agent's behavioral mode $\xi_{i}^{\tau}$. Finally, $w_{i}^{\tau}\left(\xi_{i}^{\tau}\right)$ is one of two zero-mean, stationary, Gaussian white noise processes used to model the unpredictable decision-motive processes of agent $i$. Which of the two processes that is applied to the model at a given time is determined by the behavioral mode, $\xi_{i}^{\tau}$. The two white noise signals are distinguished by different covariance matrices, and each is uncorrelated with $p_{j} \forall j$. Nonholonomic constraints of the cows are neglected in this treatment, though they could be incorporated with an increase in the complexity of the model structure. Note that the force terms are only applied to affect changes in velocity in accordance with Newton's second law.

\subsection{Agent-to-Agent Interaction Force}

Dropping the $\tau$ superscripts for clarity, the form of the agent coupling force $f_{i j}\left(p_{i}, p_{j}, \xi_{i}\right)$ is given by

$$
f_{i j}\left(p_{i}, p_{j}, \xi_{i}\right)=\left(\alpha\left(\xi_{i}\right) \theta_{1}-\frac{\theta_{2}}{\left\|p_{j}-p_{i}\right\|}\right) n_{i j},
$$

where $n_{i j}=\left(p_{j}-p_{i}\right) /\left\|p_{j}-p_{i}\right\|$ is the unit vector along the line from $p_{i}$ to $p_{j}$ (henceforth, $\|\cdot\|$ will denote the $\ell^{2}$ norm).

The parameter $\alpha\left(\xi_{i}\right)$ is used to model the animal's tendency to aggregate as a function of its behavioral mode. During grazing $\alpha\left(\xi_{i}=G\right)=1$, which corresponds to nominal behavior. When the animal is stressed, $\alpha\left(\xi_{i}=S\right)>1$, corresponds to an increased attraction to neighbors. In this work we investigate, among other things, the effects of different values of $\alpha\left(\xi_{i}=S\right)$. Whereas in [8] we calibrated parameters $\theta_{1}$ and $\theta_{2}$ specific to each cow pair, we use values for $\theta_{1}$ and $\theta_{2}$ averaged over all cow pairs in this paper, which allows us to extrapolate the models to larger herds. For illustration, (4) given by $\left\|f_{i j}\right\|=\alpha\left(\xi_{i}\right) \theta_{1}-\theta_{2} /\left\|p_{j}-p_{i}\right\|$ is shown in the right of Figure 2

\section{Experimental Setup}

We are interested in the impact of the number of controlled animals on the performance of an open-loop control algorithm that moves the herd from an initial position to a defined final goal position by using the concept of a virtual fence [5,9]. In all our experiments, the fence is modeled by a circular disc of $25 \mathrm{~m}$ diameter. The fence coordinates and their time evolution are assumed to be known to the sensor box. If the cow leaves the fenced area and is wearing an actuation device, it will be given a stimulus.

At its initial position the center is at $(0 m,-50 m)$. The center of the fence then moves with speed $v_{f}=20 \frac{\mathrm{m}}{h}$ northwards. After $5 h$ simulated time the experiment is stopped. We then measure the average number of individuals within the fence over the whole experiment as well as the final number of individuals within the fence at time $5 h$. Figure 3 illustrates the experimental setup, showing a random initial distribution 
(left), a successful final configuration (middle), and an unsuccessful final configuration (right). All simulations have been conducted with 30 cows. The covariance of the driving noise, $w_{i}^{\tau}$, during stress was set to be twice as high as during grazing, which roughly corresponds to observations from [9]. The time constant for stress decay has been set to $T=30 s$. All parameter values being used are summarized in Tab. 4

\begin{tabular}{|c|c|c|c|c|c|}
\hline$\Delta t$ & $=1 \mathrm{~s}$ & $a_{i}$ & $=.9294$ & $w_{i}\left(\xi_{i}=G\right)=$ & $\left(\begin{array}{cc}1.22 e-2 & -9 e-4 \\
-9 e-4 & 1.46 e-2\end{array}\right)$ \\
\hline$\theta_{1}$ & .0225 & $\theta_{2}$ & 732 & $w_{i}\left(\xi_{i}=S\right)=$ & $\left(\begin{array}{l}2.44 e-2-18 e-4 \\
-18 e-42.92 e-2\end{array}\right)$ \\
\hline
\end{tabular}

Table 1. Parameters used for simulating the system defined by Equations 3 and 4 .

Simulations are performed using the MATLAB Distributed Computing Toolbox on the CSAIL computational cluster. The ratio between simulated and computational time is roughly 15 on a modern 64bit workstation.

\section{Results}

As we are unaware of the effective amplification of the aggregation force $\alpha$ as well as the existence and specific values of the radius within which cows are socially affected by the behavioral mode of neighboring cows, we perform a parameter sweep over $\alpha\left(\xi_{i}=\right.$ $S)=\{1,2,4\}$ and $R=\{0,5,10\}$ for $1-10,15,20,25$ and 30 controlled cows.

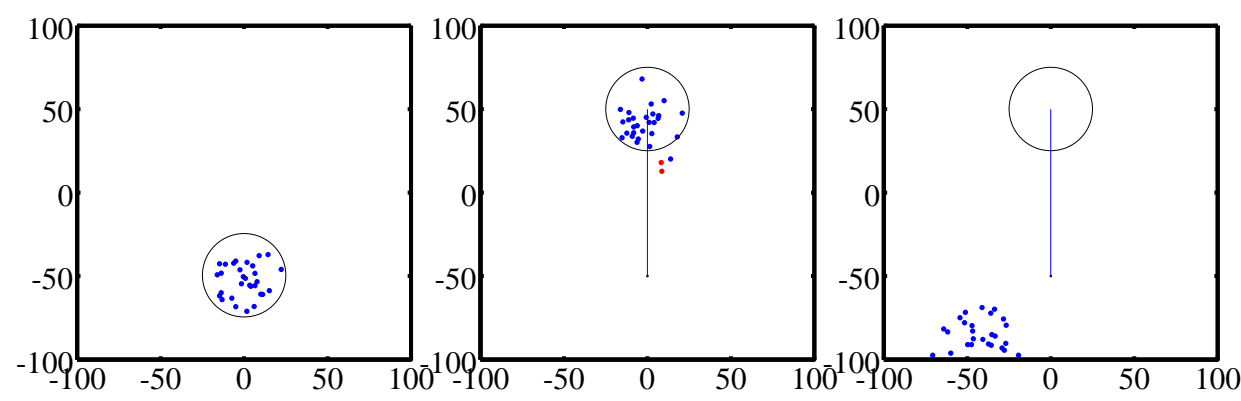

Fig. 3. Left: Random initial deployment of 30 cows and virtual fence configuration. Middle: Example of a final configuration after successful control (all animals are controlled). The herd oscillates around the center of the fenced area; red dots (cows outside the fence) denote cows being stressed. Right: Example of unsuccessful control (no animals were controlled). The herd does a random walk around the center of its initial deployment area.

Figure 4, left, shows the average number of individuals within the fence over the whole experiment for $\alpha=2$, i.e. the attraction force between cows is twice as high during stress when compared with grazing, and different values of $R$ for 50 simulations 
per data point ( 2250 simulations in total). Figure 4 , right, shows the ratio of experiments where more than 15 cows where within the fence after $5 \mathrm{~h}$. These results confirm that manipulating 10 of 30 cows is sufficient for moving to a desired location using a virtual fence, assuming that the aggregation forces are twice as large during stress as during aggregation and that social amplification exists for a radius of $R=5 \mathrm{~m}$. For $R=10 \mathrm{~m}$, however, the controller performance decreases. This can be explained by the fact that, for a large radius, the whole herd tends to be in the same mode, inhibiting the controlling action of the virtual fence. For example, if most animals are unstressed, a large number of unstressed neighbors are always within the radius of any given cow, thereby outnumbering the stressed neighbors and inhibiting the propagation of stress. Conversely, if there is a large number of stressed animals, it is likely that the whole herd becomes stressed and the virtual fence is rendered ineffective as crossing the fenceline will make no difference as the animals are already stressed. We refer to this effect as a "stampede" since it bears an obvious resemblance to that phenomenon in natural herds.
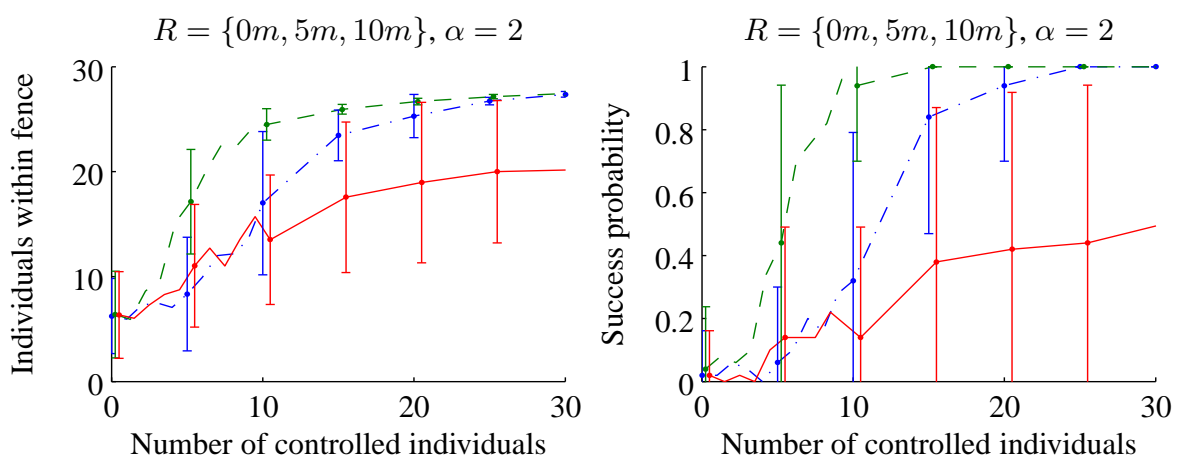

Fig. 4. Left: Average number of individuals within the fenced area during $5 h$ of simulated time for $R=0,5,10\left(-,,--,-/\right.$ blue, green, red) with $\alpha\left(\xi_{i}=S\right)=2$ vs. the number of controlled individuals. Without assuming propagation of stress $(R=0)$ we observe a significant increase in performance for 10 controlled individuals. Right: Ratio of successful to unsuccessful simulation runs (success is defined as $>50 \%$ of the animals within the fence at the final fence location). 50 experiments per data-point, error bars are standard deviation.

We were then interested in testing the influence of the presumed increase in gregarious behavior for stressed animals. We tested two extreme cases: $\alpha\left(\xi_{i}=S\right)=1$, so that stressed animals experience no greater attraction to their neighbors than grazing animals, and $\alpha\left(\xi_{i}=S\right)=4$, so that stressed animals experience four times the attraction to their neighbors. Results for various values of $R$ are shown in Fig. 5 for both of these cases. We clearly see that $\alpha\left(\xi_{i}=S\right)>1$ is a necessary condition for social herding, and is independent of the number of artificially modified individuals, as well as potential social amplification. We also observe that social amplification seems to become less important for high values of $\alpha\left(\xi_{i}=S\right)$.

We are also interested in the impact of the time constant of stress $T$ and the size of the driving noise covariance matrix. We therefore ran simulations for $\alpha\left(\xi_{i}=S\right)=2$ 

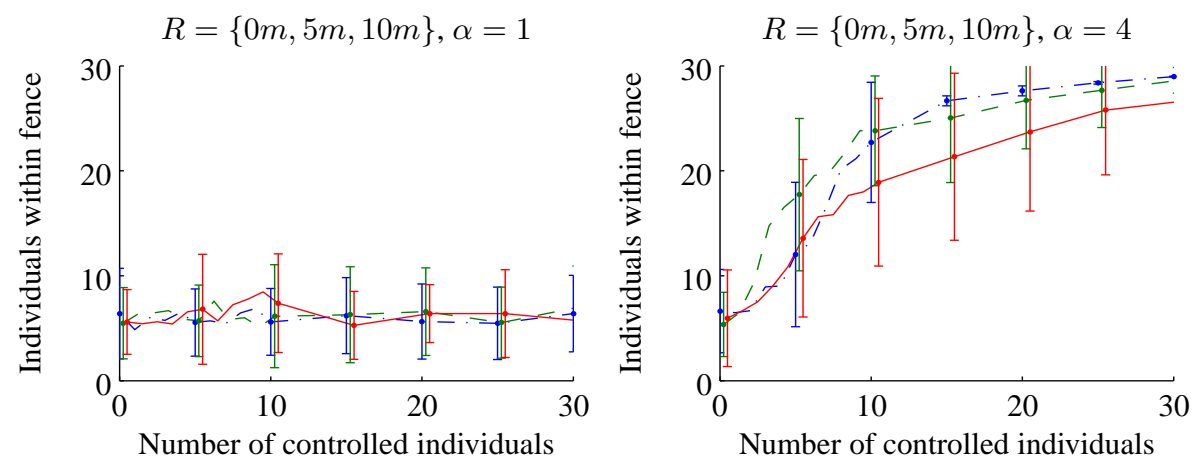

Fig. 5. Average number of individuals within the fenced area for the same experimental conditions as in Figure 4 but with $\alpha\left(\xi_{i}=S\right)=1$ and $\alpha\left(\xi_{i}=S\right)=4$ (left and right plot). For $\alpha\left(\xi_{i}=\right.$ $S)=1$, herd control fails (left). 50 experiments per data-point, error bars are standard deviation.

and $R=5$ for $T=\{1 s, 30 s, 60 s\}$ and multiplied the covariance matrix of the noise applied to the agents by 1, 2 or 4. Results for various $T$ are shown in Fig. 6, left. We observe that stress indeed needs to be maintained for some time after the stimulus as $T=1$ yields poor performance. Although we did not explicitly test larger values than 60 s for $T$, we conjecture that high values of $T$ will eventually lead to all animals being in the stressed mode, which will cause the fence to be ineffectual. With respect to the driving noise covariance, we do not observe any significant difference in performance for the values that we tried.

Finally, we are interested how social control scales and experimented with the ratio of manipulated animals in team sizes of 10, 30 and 100 animals (Figure 6, right). Results show that modifying only a part of the herd becomes increasinlgy efficient for larger herds, and that controlling as little as $20 \%$ of the animals might be sufficient for the fence geometry and speed being chosen. Whereas performance for 30 and 100 animals is similar, control of herds with only 10 animals seems to be generally more difficult.

\section{Discussion}

Our results show the potential for social control of a cow herd given that a) the gregarious behavior increases under stress, and b) stress propagates through the herd by mutual observation. While further field experiments will allow us to better understand the differences in the proposed behavioral modes, we argue that the gregarious behavior might be stimulated to the necessary extent by providing directional impulses to the cows, e.g. using the sound system available on our sensor box. In this case, results from Fig. 5 suggest that high levels of gregarious behavior, be it innate to the species or artificially stimulated, can achieve the same level of performance (in terms of the number of modified individuals required) as a system with weak gregarious behavior but social amplification of stress.

In this paper, our model assumes that attraction and repulsion forces are the same for every neighbor. Results from [8] and observations on feral cattle [10] suggest, how- 

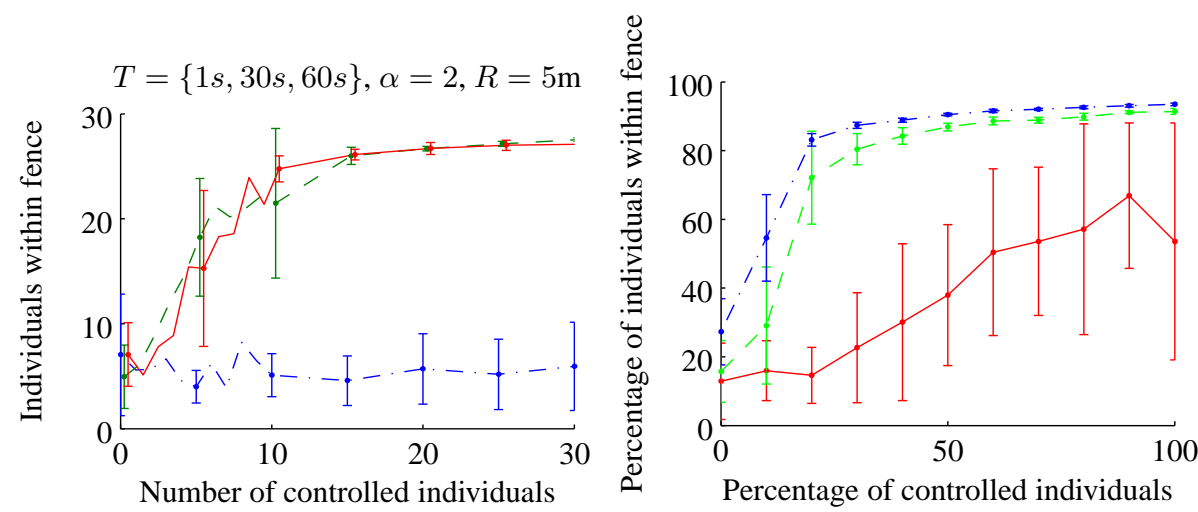

Fig. 6. Left: Average number of individuals within the fenced area for the same experimental conditions as in Figure 4, but for $T=\{1,30,60\}$ (- .,--,- / blue, green, red). Right: Percentage of individuals within fence vs. percentage of controlled individuals for team sizes of 10,30, and 100 animals (-,--,-. / red, green, blue). 10 simulations per data point.

ever, the existence of social preferences among the cows. Depending on the strength of such preferences, modifying a specific subset of the herd might increase controller performance. While kinship is usually well known in a domestic herd, further studies might reveal a relation between phenomenological properties and social leadership. It would then seem beneficial to apply stimuli to known social leaders. In [9] also spatiotemporal preferences within the herd (e.g. shadow seeking behavior or specific habits) are observed. Although such preferences might jeopardize an open-loop control approach, they might also be exploited for more effective control by planning trajectories, which are easily followed by the herd.

\section{Conclusion}

We extended the dynamical model for a cow herd presented in [8] by a hybrid structure, which differentiates between a grazing and a stressed behavioral mode. Using this model, we show that open-loop control of the cow herd using virtual fences is possible if the gregarious behavior is sufficiently stronger during stress than during grazing. Control of the herd exploits the natural gregarious behavior of the animal and does not require any learning of stimulus/action patterns. Moreover, we showed that when stress is propagated to neighbors (social amplification), robust control can be achieved by endowing only a fraction of animals with sensing and actuation.

Although we did not calibrate parameters for the animal behavior during stress, systematic simulations of our model suggest that driving noise covariance and stress decay-time are of little importance for the performance of social control, whereas an increase in gregarious behavior and stress propagation within the herd seem to be of utmost importance. In the future, we plan to artificially induce stress using aural signals of varying strength. We would then like to quantitatively validate the existence of distinct behavioral modes (grazing and stressed) and quantify its parameters. Specifically, we are interested in the increase in gregarious behavior and the mechanisms of social 
amplification. Given such an enhanced model for this particular species, we would then like to generalize the methodology to other gregarious animals and develop closed-loop control schemes for robust, low-stress control of farm animals.

\section{Acknowledgments}

The authors would like to thank Dean Anderson, Carrick Detweiler, and Iuliu Vasilescu for providing the animal data. N. Correll is sponsored by a grant from the Swiss National Science Foundation under contract number PBEL2-118737. M. Schwager was supported in part by NSF grant IIS-0513628 and the MURI SWARMS project.

\section{References}

1. R. Vaughan, N. Sumpter, J. Henderson, A. Frost, and S. Cameron, "Robot control of animal flocks," in Proceedings of the 1998 IEEE International Symposium on Intelligent Control (ISIC). IEEE Press, Piscataway, New Jersey, USA, 1998, pp. 277-282.

2. T. Quigley, H. Sanderson, A. Tiedemann, and M. McInnis, "Livestock control with electrical and audio stimulation," Rangelands, June 1990.

3. A. Tiedemann, T. Quiqley, L. White, W. Lauritzen, J. Thomas, and M. McInnis, "Electronic (fenceless) control of livestock," United States Department of Agriculture, Forest Service, Tech. Rep. PNW-RP-510, January 1999.

4. D. Anderson and C. Hale, "Animal control system using global positioning and instrumental animal conditioning," US Deparment of Agriculture, Tech. Rep. US Patent 6,232,880, May 2001

5. D. Anderson, "Virtual fencing - past, present and future," The Rangeland Journal, vol. 29, pp. 65-78, 2007.

6. J. Halloy, J.-M. Amé, G. S. C. Detrain, G. Caprari, M. Asadpour, N. Correll, A. Martinoli, F. Mondada, R. Siegwart, and J.-L. Deneubourg, "Social integration of robots in groups of cockroaches to control self-organized choice," Science, vol. 318, no. 5853, pp. 1155-1158, 2007.

7. G. Caprari, A. Colot, R. Siegwart, J. Halloy, and J.-L. Deneubourg, "Building mixed societies of animals and robots," IEEE Robotics \& Automation Magazine, vol. 12, no. 2, pp. 58-65, 2005.

8. M. Schwager, C. Detweiler, I. Vasilescu, D. Anderson, and D. Rus, "Data-driven identification of group dynamics for motion prediction and control," Journal of Field Robotics, 2008, to appear.

9. Z. Butler, P. Corke, R. Peterson, and D. Rus, "From robots to animals: Virtual fences for controlling cattle,” I. J. Robotic Res., vol. 25, no. 5-6, pp. 485-508, 2006.

10. A. Lazo, "Social segregation and the maintenance of social stability in a feral cattle population," Animal Behavior, vol. 48, pp. 1133-1141, 1994. 